Research Article

\title{
Patient Delay in Hospital Visiting and the Weekend Effect of Surveillance Report on Hand-Foot-and-Mouth Disease and Epidemic Parotitis in Hanzhong City, China
}

\author{
Jianjun Wei, ${ }^{1}$ Zhonghai Zhu, ${ }^{2}$ Qi Qi, ${ }^{2}$ and Lingxia Zeng $\mathbb{D}^{2}$ \\ ${ }^{1}$ Center for Disease Control and Prevention, Hanzhong, Shaanxi, China \\ ${ }^{2}$ School of Public Health, Xi'an Jiaotong University Health Science Center, Shaanxi 710061, China \\ Correspondence should be addressed to Lingxia Zeng; tjzlx@mail.xjtu.edu.cn
}

Received 23 October 2019; Revised 19 February 2020; Accepted 21 April 2020; Published 6 May 2020

Academic Editor: Maria Lina Tornesello

Copyright (c) 2020 Jianjun Wei et al. This is an open access article distributed under the Creative Commons Attribution License, which permits unrestricted use, distribution, and reproduction in any medium, provided the original work is properly cited.

Background. We aimed at investigating the prevalence and associated factors of patient delay in hospital visiting and weekend effect of disease surveillance on hand-foot-and-mouth disease and epidemic parotitis/mumps. Methods. Daily report data on hand-foot-and-mouth disease and epidemic parotitis cases between January 1, 2014, and December 31, 2017, in Hanzhong, Shaanxi, China, were collected. The patient delay in hospital visiting was defined by the date difference between disease onset and patient's visit to hospital. Differences of delayed durations and percentages were compared by using nonparametric or $\chi^{2}$ tests across gender, age, occupation, disease classification, epidemic and nonepidemic seasons, and years of disease onset. Additionally, to determine whether there existed a weekend effect of disease surveillance, the mean cases reported on weekdays and weekends were also compared. Results. A total of 14,814 patients with hand-foot-and-mouth disease and 4013 with epidemic parotitis were recorded, respectively. We found that $43.1 \%$ of the hand-foot-and-mouth disease and $36.5 \%$ of the epidemic parotitis patients had delayed visiting to hospital. All patients were reported through the online surveillance system on the day of visiting hospital. The percentage of delayed visiting to hospital differed significantly by years and epidemic and nonepidemic seasons and between children in and not in childcare center (all $p$ values $<0.05$ ). In addition, the reported numbers of both diseases fluctuated on weekdays but obviously decreased on weekends regardless of the epidemic or nonepidemic seasons. Conclusions. The reported cases of HFMD and epidemic parotitis had an obvious weekend effect, with an increasing tendency of cases delaying in hospital visiting over the recent years in Hanzhong, China. Parents and caregivers rather than health systems should be primarily targeted for the prevention and control of infectious diseases and their local outbreaks such as community-based education on the seconddose vaccination of mumps and/or hand hygiene.

\section{Background}

Epidemic parotitis/mumps is an acute infectious disease of the respiratory tract and characterized by a highly recessive infection rate and long infectious durations [1]. Patients in the early stage or recessively infected individuals are not easily identified resulting in a source of infection and may easily cause outbreaks or epidemic in collective units, such as schools and childcare centers [2]. China has included it in the disease surveillance information report management system since 2004 [3]. The reported incidence of epidemic parotitis between 2004 and 2010 in China fluctuated around
20/100,000 [4]. However, in 2008 and 2009, epidemic parotitis outbreaks accounted for approximately $21 \%$ of all outburst epidemics of infectious diseases in China, which increased to 34\% in 2010 and 2012 [5-7]. In addition, students and children are the targeted populations of epidemic parotitis $[3,5,6]$, accounting for approximately $93 \%$ of all cases each year. Epidemic parotitis outbreaks in schools took up approximately $99 \%$ of all epidemic parotitis outbreaks. Since 2004, the incidence of epidemic parotitis in China has been highest among children $<15$ years, which accounted for approximately $85 \%$ to $92 \%$ of all cases $[3,5,6]$. Among these children cases, the incidence was highest in 
children between 5 and 9 years, accounting for approximately $43 \%$ to $49 \%$ of all cases [8]. Key factors causing the constant epidemic include an insensitive surveillance system, improper implementation of relevant policies, and an inability to provide a timely diagnosis and reporting and effective quarantine for patients at the beginning of the disease onset [4].

Recently, the hand-foot-and-mouth disease (HFMD) epidemic in China has shown characteristics of a fairly high incidence rate and long peak durations and wide distribution of the epidemic [9]. After 2008, medical institutes in each level in China were required to report the HFMD cases directly through the online disease surveillance information report management system according to the "Law of the People's Republic of China on the prevention and treatment of infectious disease." Cases with HFMD from children under 5 years of age constituted more than $80 \%$ of all cases with the highest incidence among children between 1 and 3 years $[10,11]$. The most effective strategy to prevent the spread of HFMD is to strengthen surveillance reporting system of the disease epidemic with a focus on active surveillance of children under 5 years of age and ensure daily monitoring and reporting, in which patients would be identified, diagnosed, reported, quarantined, and treated as early as possible [12]. However, analyses on surveillance data from multiple locations showed a significant weekend effect on HFMD reporting [13, 14].

Because the two infectious diseases discussed above mainly target on infants and school-aged children, the surveillance quality, timeliness, and completeness of routine surveillance have great implications for follow-up managements of cases and health departments to prevent the incidence and even outbreaks of these infectious diseases. Along with the improvement of the direct online reporting system, the time difference between the disease diagnosis and recording formation in the online report system, i.e., diagnosis to report, usually is within one day in China. However, the time difference between disease onset and diagnosis at hospitals, i.e., onset to diagnosis, is an emerging issue that compromised the timely report of infectious diseases on the online reporting system $[15,16]$. Given that these two infectious diseases easily developed into outbreaks, we hypothesized the time difference between disease onset and diagnosis and the potential weekend effect of epidemic reporting are probably the primary issues that affect the quality of infectious disease surveillance.

Based on daily report data for HFMD and epidemic parotitis cases between January 1, 2014, and December 31, 2017, in Hanzhong, Shaanxi, China, we aimed at investigating the prevalence and influencing factors of patient delay in hospital visiting and the possibility of a weekend effect of disease surveillance. We also aimed at comparing the durations of delay in hospital visiting for these two diseases between epidemic and nonepidemic seasons, which would provide evidence for the improvement of the surveillance quality and the control of the spread of these two diseases among young children.

\section{Methods}

2.1. Data Source. Data on HFMD and epidemic parotitis were obtained from the "Infectious disease report information management system" subsystem of the "China disease prevention and control information system" between January 1, 2014, and December 31, 2017. According to the regulations stated in the "Law of the People's Republic of China on the prevention and treatment of infectious disease" and "Standard for the management of infectious disease information report," the first physician of receiving patients at every medical institution should fill out the online "Infectious disease report card of the People's Republic of China" once the patient was diagnosed with an infectious disease. The following information was collected including the patients' gender, date of birth/ age, race, occupation, residential address, classification of diseases (clinically diagnosed cases and confirmed cases), and date of disease onset, diagnosis, and online report.

\subsection{Diagnostic Criteria of HFMD and Epidemic Parotitis.} HFMD was defined by the "Diagnosis and treatment guidelines of HFMD (2010 edition)" that was published by the Ministry of Health on April 27, 2010 [17]. Clinically diagnosed cases were patients who had disease onset during the epidemic season and usually were preschool children and infants. The common symptoms included fever and rash on the hands, feet, mouth, and hip. Clinically diagnosed cases were further confirmed as confirmed cases with the presence of any one of the following criteria: (1) a positive nucleic acid test of enterovirus, e.g., coxsackievirus A 16 (CoxA16) and enterovirus71 (EV71), (2) isolated enteroviruses that were identified to be CoxA16, EV71, or other enteroviruses that might cause HFMD, and (3) the serum antibody titers of CoxA16, EV71, or other enteroviruses that might cause HFMD increased more than 4-fold during the acute and recovery phases.

The diagnostic criteria for epidemic parotitis were based on the "Epidemic parotitis diagnostic criteria, WS270-2007" which was approved by the Ministry of Health, People's Republic of China, and issued on October 15, 2007 [18]. Clinically diagnosed cases were defined using the following criteria: (1) swelling, pain, and/or amplifying pain of the unilateral, bilateral parotid glands or other saliva glands during mouth opening, chewing, and eating acidic food, (2) symptoms like fever, headache, fatigue, and loss of appetite, (3) contacting with individuals infected by epidemic parotitis 14 to 28 days before disease onset, and/or those living in a region with an ongoing epidemic parotitis and having symptoms like fever, headache, fatigue, and loss of appetite, and (4) elevated serum and urine amylase levels and/or changes of cerebral spinal fluid indicating the presence of viral meningitis. Laboratory-confirmed cases were further identified from clinically confirmed cases by the following criteria: (1) elevated epidemic parotitis virus-specific IgM antibodies in the serum, (2) a 4-fold or higher elevation of epidemic parotitis virus-specific IgG antibody titers during the recovery or acute phases, and/or (3) isolated epidemic parotitis virus from body fluids, such as the saliva, urine, and cerebral spinal fluid. 
2.3. Interest of Indicators and Definitions. We documented the earliest date of occurrence of relevant symptoms based on patient self-reporting as date of disease onset. Date of patient visiting hospital was the date when the patient firstly visited the hospital. Report date referred to the date when diseases were reported through the online disease surveillance/prevention and control system. Delayed hospital visiting was defined by the durations between the patient visiting date and the disease onset date over one day. Epidemic season of HFMD was defined from April to July and from November to December of each year [6], while the rest months of the year were considered as nonepidemic season. Similarly, the epidemic season for epidemic parotitis included April to July of each year and November to January of the following year, and the nonepidemic seasons included February to March and August to October of each year [6]. In addition, in the present study, the term "weekend" referred to both "natural weekend" and "nonnatural weekend" days, i.e., official festival or holiday break due to New Year's Day, Spring Festival, Qingming Festival, Labor Day, Dragon Boat Festival, Mid-Autumn Festival, and National Day in China. The holiday information was obtained from the "Notice on part of holiday arrangements" issued by the General Office of the State Council of the People's Republic of China each year. The day before the statutory holiday was defined as "Friday," and the first return work day after each holiday was defined as "Monday." The daily average numbers of cases reported on weekend referred to days of Saturday and Sunday defined above.

2.4. Statistical Analysis. The durations of patient delay in hospital visiting, i.e., 0-1 day, 2-3 days, $4-7$ days, and $>7$ days, were described as frequencies and percentages, and the differences across factors were further compared using nonparametric tests. The odds ratio (OR) and its 95\% confidence interval (CI) of these factors with the binary delayed hospital visiting were also estimated using multivariable logistic regression. The average number of daily disease onsets and reported cases on workdays and weekends were represented as the mean \pm standard deviation, respectively.

A two-side $p<0.05$ was regarded as statistical significance. All statistical analysis was performed using SPSS v.18.0 (IBM Corp., Armonk, NY, USA).

\section{Results}

3.1. Background Information of Included Patients. A total of 14,814 patients with HFMD were reported between January 1, 2014, and December 31, 2017, in Hanzhong, China. Among whom, 96.6\% were clinically diagnosed cases, 96.3\% of patients were children in or not in childcare center, and $88.0 \%$ were younger than five years of age (Table 1). Regarding epidemic parotitis, 4013 patients were recorded, among whom $99.2 \%$ were clinically diagnosed cases, $63.8 \%$ were students, and $52.7 \%$ were between 5 and 10 years of age (Table 1).
3.2. Durations of Delay between Disease Onset and Hospital Visiting. $43.1 \%$ of patients with HFMD and $36.5 \%$ of patients with epidemic parotitis experienced delayed hospital visiting after disease onset. The percentages of durations of delay between disease onset and hospital visiting at 2-3, 4-7, and $>7$ days were $34.1 \%, 7.7 \%$, and $1.3 \%$ for HFMD, respectively. The corresponding percentages for epidemic parotitis were $24.3 \%, 9.8 \%$, and $2.3 \%$, respectively. In addition, all patients were reported through the online disease surveillance system on the day when visiting the hospital.

\subsubsection{Factors Associated with Patient Delay in Hospital} Visiting. The durations of delayed hospital visiting of both diseases differed significantly by years, ages, occupations, and epidemic seasons (Tables 2 and 3).

In the multivariable logistic models (Table 4), we found that the risks of delayed hospital visiting for HFMD in 2015, 2016, or 2017 were significantly higher as compared with that in 2014. The adjusted OR was 1.35 (95\% CI: 1.23, 1.48), 1.16 (95\% CI: $1.06,1.27)$, and 1.16 (95\% CI: $1.05,1.27)$, respectively. Compared to children in childcare center, the risk of delayed hospital visiting increased by $41.2 \%$ (95\% CI: $30.2 \%, 53.2 \%)$ and $32.2 \%$ (95\% CI: 6.8\%, 63.7\%) among children who were not in childcare center and school students, respectively. We also found that the risk of occurring delayed hospital visiting in epidemic season was significantly lower than that in nonepidemic season with an adjusted OR of 0.78 (95\% CI: $0.72,0.85$ ).

In terms of epidemic parotitis, the percentages of delayed hospital visiting increased significantly by years, ranging from $27.9 \%$ in 2014 to $40.1 \%$ in 2017 . In addition, the risk of occurring delayed hospital visiting in epidemic season reduced by $15.1 \%$ (95\% CI: 3.8\%, 25.8\%) when compared with nonepidemic season.

3.3. Distribution of Case Numbers and Reported Numbers within the Week. No obvious pattern of disease onset time was identified for HFMD or epidemic parotitis, and no weekend effect was detected. In contrast, the reported numbers of HFMD and epidemic parotitis cases both showed weekly patterns either in the epidemic or in the nonepidemic season, which decreased from Monday to the weekend with uniformly lower numbers on weekends than that on Monday (Figures 1 and 2, Supplementary Tables 1 and 2). Besides, the statistical tests to determine the difference of difference (DID) of reported and actual cases between weekend and weekday showed that the number of reported cases on weekends was uniformly and significantly lower than that of actual cases during weekdays. The mean (SD) of DID was 0.79 (3.27) for epidemic parotitis and 1.44 (3.31) for hand-foot-and-mouth disease on weekends, with corresponding $p$ values of $<0.001$ and $<0.001$, respectively.

\section{Discussion}

4.1. Main Findings. We found that $88.0 \%$ of HFMD cases were children younger than 5 years of age between 2014 and 2017. In contrast, the majority of the patients with epidemic 
TABLE 1: Background information of patients with hand-foot-and-mouth disease or epidemic parotitis.

\begin{tabular}{lcc}
\hline Items & Hand-foot-and-mouth disease $(n(\%))$ & Epidemic parotitis $(n(\%))$ \\
\hline Year & & \\
2014 & $5067(34.2)$ & $774(19.3)$ \\
2015 & $3183(21.5)$ & $548(13.7)$ \\
2016 & $3650(24.6)$ & $1020(25.7)$ \\
2017 & $2914(19.7)$ & $1671(41.6)$ \\
\hline Gender & & $2266(56.5)$ \\
Male & $8696(58.7)$ & $1747(43.5)$ \\
Female & $6118(41.3)$ & $3981(99.2)$ \\
\hline Classification of disease & & $32(0.8)$ \\
Clinically diagnosed cases & $14,311(96.6)$ & \\
Confirmed cases & $503(3.4)$ & $664(16.5)$ \\
\hline Occupation & & $255(6.4)$ \\
Children in childcare & $4420(29.8)$ & $2561(63.8)$ \\
Children not in childcare & $9706(65.5)$ & $407(10.1)$ \\
Students & $632(4.3)$ & $126(3.1)$ \\
Peasants & $29(0.2)$ & \\
Others & $27(0.2)$ & $8(0.2)$ \\
\hline Age & & $512(12.8)$ \\
$\quad<1$ year & $1592(10.7)$ & $2115(52.7)$ \\
$1-4$ years & $11,457(77.3)$ & $1378(34.3)$ \\
5-10 years & $1585(10.7)$ & \\
>10 years & $180(1.2)$ & \\
\hline
\end{tabular}

TABLE 2: Comparisons of durations of delay in patient hospital visiting for hand-foot-and-mouth disease across different factors.

\begin{tabular}{|c|c|c|c|c|c|c|}
\hline \multirow{2}{*}{ Factors } & \multicolumn{4}{|c|}{ Durations of delay in patient hospital visiting (days) } & \multirow{2}{*}{ Statistics } & \multirow{2}{*}{$p$ values } \\
\hline & $0-1$ & $2-3$ & $4-7$ & $>7$ & & \\
\hline Year & & & & & 90.532 & $<0.001$ \\
\hline 2014 & $3077(60.7)$ & $1569(31.0)$ & $361(7.1)$ & $60(1.2)$ & & \\
\hline 2015 & $1679(52.7)$ & $1210(38.0)$ & $274(8.6)$ & $20(0.6)$ & & \\
\hline 2016 & $2022(55.4)$ & $1242(34.0)$ & $323(8.8)$ & $63(1.7)$ & & \\
\hline 2017 & $1649(56.6)$ & $1032(35.4)$ & $188(6.5)$ & $45(1.5)$ & & \\
\hline Gender & & & & & 3.458 & 0.326 \\
\hline Male & $4968(57.1)$ & $2974(34.2)$ & $643(7.4)$ & $111(1.3)$ & & \\
\hline Female & $3459(56.5)$ & $2079(34.0)$ & $503(8.2)$ & $77(1.3)$ & & \\
\hline Classification of disease & & & & & 300.086 & $<0.001$ \\
\hline Clinically diagnosed cases & $8157(57.0)$ & $4897(34.2)$ & $1118(7.8)$ & $139(1.0)$ & & \\
\hline Confirmed cases & $270(53.7)$ & $156(31.0)$ & $28(5.6)$ & $49(9.7)$ & & \\
\hline Occupation & & & & & 133.591 & $<0.001$ \\
\hline Children in childcare & $2799(63.3)$ & $1335(30.2)$ & $252(5.7)$ & $34(0.8)$ & & \\
\hline Children not in childcare & $5269(54.3)$ & $3459(35.6)$ & $831(8.6)$ & $147(1.5)$ & & \\
\hline Students & $340(53.8)$ & $231(36.6)$ & $54(8.5)$ & $7(1.1)$ & & \\
\hline Peasants and others & $19(33.9)$ & $28(50.0)$ & $9(16.1)$ & $0(0.0)$ & & \\
\hline Age & & & & & 45.480 & $<0.001$ \\
\hline$<1$ year & $857(53.8)$ & $586(36.8)$ & $126(7.9)$ & $23(1.4)$ & & \\
\hline $1-4$ years & $6533(57.0)$ & $3890(34.0)$ & $883(7.7)$ & $151(1.3)$ & & \\
\hline $5-10$ years & $967(61.0)$ & $488(30.8)$ & $117(7.4)$ & $13(0.8)$ & & \\
\hline$>10$ years & $70(38.9)$ & $89(49.4)$ & $20(11.1)$ & $1(0.6)$ & & \\
\hline Epidemic season & & & & & 56.490 & $<0.001$ \\
\hline Yes & $6821(58.3)$ & $3913(33.4)$ & $842(7.2)$ & $132(1.1)$ & & \\
\hline No & $1606(51.7)$ & $1140(36.7)$ & $304(9.8)$ & $56(1.8)$ & & \\
\hline
\end{tabular}

parotitis were school students $(63.8 \%)$, with children aged 5-10 years accounting for $52.7 \%$. We also found that $43.1 \%$ of patients with HFMD and $36.5 \%$ of patients with epidemic parotitis had delays in hospital visiting in nonepidemic seasons, which was slightly reduced, but similar issue still identified in epidemic seasons. Majority of the durations of delaying hospital visiting lied within two to three days after disease onset. Besides, the risk of delays in hospital visiting for epidemic parotitis showed an increasing trend with the years. We did not identify the weekend effect on the time of disease onset for both the diseases above. However, we found an obvious weekend effect on the time of online reporting 
TABLE 3: Comparisons of durations of delay in patient hospital visiting for epidemic parotitis across different factors.

\begin{tabular}{|c|c|c|c|c|c|c|}
\hline \multirow{2}{*}{ Factors } & \multicolumn{4}{|c|}{ Durations of delay in patient hospital visiting (days) } & \multirow{2}{*}{ Statistics } & \multirow{2}{*}{$p$ values } \\
\hline & $0-1$ & $2-3$ & $4-7$ & $>7$ & & \\
\hline Year & & & & & 48.823 & $<0.001$ \\
\hline 2014 & $558(72.1)$ & $159(20.5)$ & $44(5.7)$ & $13(1.7)$ & & \\
\hline 2015 & $356(65.0)$ & $142(25.9)$ & $42(7.7)$ & $8(1.5)$ & & \\
\hline 2016 & $635(62.3)$ & $252(24.7)$ & $104(10.2)$ & $29(2.8)$ & & \\
\hline 2017 & $1001(59.9)$ & $424(25.4)$ & $202(12.1)$ & $44(2.6)$ & & \\
\hline Gender & & & & & 1.749 & 0.626 \\
\hline Male & $1439(63.5)$ & $541(23.9)$ & $229(10.1)$ & $57(2.5)$ & & \\
\hline Female & $1111(63.6)$ & $436(25.0)$ & $163(9.3)$ & $37(2.1)$ & & \\
\hline Classification of disease & & & & & 3.089 & 0.378 \\
\hline Clinically diagnosed cases & $2533(63.6)$ & $968(24.3)$ & $388(9.7)$ & $92(2.3)$ & & \\
\hline Confirmed cases & $17(53.1)$ & $9(28.1)$ & $4(12.5)$ & $2(6.2)$ & & \\
\hline Occupation & & & & & 22.149 & 0.008 \\
\hline Children in childcare & $455(68.5)$ & $150(22.6)$ & $50(7.5)$ & $9(1.4)$ & & \\
\hline Children not in childcare & $165(64.7)$ & $65(25.5)$ & $19(7.5)$ & $6(2.4)$ & & \\
\hline Students & $1611(62.9)$ & $623(24.3)$ & $270(10.5)$ & $57(2.2)$ & & \\
\hline Peasants and others & $319(59.8)$ & $139(26.1)$ & $53(9.9)$ & $22(4.1)$ & & \\
\hline Age & & & & & 22.431 & 0.008 \\
\hline$<1$ year & $4(50.0)$ & $2(25.0)$ & $2(25.0)$ & $0(0.0)$ & & \\
\hline $1-4$ years & $344(66.9)$ & $124(24.2)$ & $34(6.6)$ & $10(2.0)$ & & \\
\hline $5-10$ years & $1377(65.1)$ & $494(23.4)$ & $203(9.6)$ & $41(1.9)$ & & \\
\hline$>10$ years & $825(59.9)$ & $357(25.9)$ & $153(11.1)$ & $43(3.1)$ & & \\
\hline Epidemic season & & & & & 8.227 & 0.042 \\
\hline Yes & $1663(64.8)$ & $616(24.0)$ & $234(9.1)$ & $52(2.0)$ & & \\
\hline No & $887(61.3)$ & $361(24.9)$ & $158(10.9)$ & $42(2.9)$ & & \\
\hline
\end{tabular}

TABLE 4: Multivariable logistic regressions on associations of influencing factors with patients delayed in hospital visiting for hand-foot-andmouth disease (HFMD) and epidemic parotitis.

\begin{tabular}{|c|c|c|c|c|c|c|c|c|c|}
\hline \multirow{2}{*}{ Disease } & \multirow{2}{*}{ Factors } & \multirow{2}{*}{$n(\%)$} & \multirow{2}{*}{$B$} & \multirow{2}{*}{ SE } & \multirow{2}{*}{ Wals } & \multirow{2}{*}{ Sig. } & \multirow{2}{*}{ OR } & \multicolumn{2}{|c|}{ 95\% CI for the OR } \\
\hline & & & & & & & & Lower limit & Upper limit \\
\hline \multirow{23}{*}{ HFMD } & Year & & & & & & & & \\
\hline & 2014 & $1990(39.3)$ & & & & & Ref & & \\
\hline & 2015 & $1504(47.3)$ & 0.298 & 0.046 & 41.449 & $<0.001$ & 1.347 & 1.230 & 1.475 \\
\hline & 2016 & $1628(44.6)$ & 0.147 & 0.045 & 10.681 & 0.001 & 1.158 & 1.061 & 1.265 \\
\hline & 2017 & $1265(43.4)$ & 0.145 & 0.048 & 9.254 & 0.002 & 1.156 & 1.053 & 1.270 \\
\hline & Gender & & & & & & & & \\
\hline & Male & $3728(42.9)$ & & & & & Ref & & \\
\hline & Female & $2659(43.5)$ & 0.025 & 0.034 & 0.525 & 0.469 & 1.025 & 0.959 & 1.096 \\
\hline & Classification of disease & & & & & & & & \\
\hline & Clinically diagnosed cases & $6154(43.0)$ & -0.171 & 0.092 & 3.445 & 0.063 & 0.843 & 0.704 & 1.010 \\
\hline & Confirmed cases & $233(46.3)$ & & & & & Ref & & \\
\hline & Occupation & & & & & & & & \\
\hline & Children in childcare & $1621(36.7)$ & & & & & Ref & & \\
\hline & Children not in childcare & $4437(45.7)$ & 0.345 & 0.042 & 69.044 & $<0.001$ & 1.412 & 1.302 & 1.532 \\
\hline & Students & $292(46.2)$ & 0.279 & 0.109 & 6.595 & 0.010 & 1.322 & 1.068 & 1.637 \\
\hline & $\begin{array}{l}\text { Peasants and others } \\
\text { Age }\end{array}$ & $37(66.1)$ & 0.513 & 0.354 & 2.099 & 0.147 & 1.671 & 0.834 & 3.346 \\
\hline & $<1$ year & $735(46.2)$ & & & & & Ref & & \\
\hline & $1-4$ years & $4924(43.0)$ & -0.009 & 0.055 & 0.035 & 0.876 & 0.991 & 0.890 & 1.105 \\
\hline & $5-10$ years & $618(39.0)$ & 0.001 & 0.089 & 0.001 & 0.995 & 1.001 & 0.841 & 1.191 \\
\hline & $>10$ years & $110(61.1)$ & 0.640 & 0.222 & 8.324 & 0.004 & 1.897 & 1.228 & 2.931 \\
\hline & Epidemic season & & & & & & & & \\
\hline & Yes & $4887(41.7)$ & -0.246 & 0.042 & 35.122 & $<0.001$ & 0.782 & 0.721 & 0.848 \\
\hline & No & $1500(48.3)$ & & & & & Ref & & \\
\hline
\end{tabular}


TABle 4: Continued.

\begin{tabular}{|c|c|c|c|c|c|c|c|c|c|}
\hline \multirow{2}{*}{ Disease } & \multirow{2}{*}{ Factors } & \multirow{2}{*}{$n(\%)$} & \multirow{2}{*}{$B$} & \multirow{2}{*}{ SE } & \multirow{2}{*}{ Wals } & \multirow{2}{*}{ Sig. } & \multirow{2}{*}{ OR } & \multicolumn{2}{|c|}{$95 \%$ CI for the OR } \\
\hline & & & & & & & & Lower limit & Upper limit \\
\hline \multirow{23}{*}{ Epidemic parotitis } & Year & & & & & & & & \\
\hline & 2014 & $216(27.9)$ & & & & & Ref & & \\
\hline & 2015 & $192(35.0)$ & 0.351 & 0.121 & 8.423 & 0.004 & 1.420 & 1.121 & 1.800 \\
\hline & 2016 & $385(37.7)$ & 0.483 & 0.104 & 21.458 & $<0.001$ & 1.621 & 1.322 & 1.989 \\
\hline & 2017 & $670(40.1)$ & 0.556 & 0.096 & 33.708 & $<0.001$ & 1.743 & 1.445 & 2.102 \\
\hline & Gender & & & & & & & & \\
\hline & Male & $827(36.5)$ & & & & & Ref & & \\
\hline & Female & $636(36.4)$ & -0.033 & 0.068 & 0.234 & 0.628 & 0.968 & 0.848 & 1.105 \\
\hline & Classification of disease & & & & & & & & \\
\hline & Clinically diagnosed cases & $1448(36.4)$ & -0.392 & 0.359 & 1.193 & 0.275 & 0.676 & 0.334 & 1.365 \\
\hline & Confirmed cases & $15(46.9)$ & & & & & Ref & & \\
\hline & Occupation & & & & & & & & \\
\hline & Children in childcare & $209(31.5)$ & & & & & Ref & & \\
\hline & Children not in childcare & $90(35.3)$ & 0.189 & 0.172 & 1.209 & 0.272 & 1.208 & 0.863 & 1.691 \\
\hline & Students & $950(37.1)$ & 0.112 & 0.121 & 0.856 & 0.355 & 1.118 & 0.883 & 1.416 \\
\hline & $\begin{array}{c}\text { Peasants and others } \\
\text { Age }\end{array}$ & $214(40.2)$ & 0.166 & 0.166 & 0.479 & 0.489 & 1.122 & 0.810 & 1.555 \\
\hline & $<1$ year & $4(50.0)$ & & & & & Ref & & \\
\hline & $1-4$ years & $168(32.8)$ & -0.678 & 0.724 & 0.878 & 0.349 & 0.508 & 0.123 & 2.097 \\
\hline & $5-10$ years & $738(34.9)$ & -0.648 & 0.736 & 0.775 & 0.379 & 0.523 & 0.124 & 2.213 \\
\hline & $>10$ years & $553(40.1)$ & -0.426 & 0.741 & 0.331 & 0.565 & 0.653 & 0.153 & 2.790 \\
\hline & Epidemic season & & & & & & & & \\
\hline & Yes & $902(35.2)$ & -0.163 & 0.069 & 5.637 & 0.018 & 0.849 & 0.742 & 0.972 \\
\hline & No & $561(38.7)$ & & & & & Ref & & \\
\hline
\end{tabular}

with the significantly lowest number of cases reported over the weekend and the highest reported on Monday, which resulted in a phenomenon of Monday jump in case reports.

4.2. Interpretations of the Findings. Both the HFMD and epidemic parotitis were infectious diseases and included in Chinese statutory management. We found that the absolute number of mumps cases consistently increased along with years from 2014 to 2017, but we were not able to report the incidence rate. $\mathrm{Su}$ and the coauthors reported a steady incidence of mumps in China between 2004 and 2013 [19], surrounding around the rate of $22 / 100,000$, and no national report after 2013 was identified. In the present study, $52.7 \%$ and $34.3 \%$ of the mumps cases were identified as school students and aged 5 to 10 and above 10 years old, respectively, which was similar to the national report $[19,20]$, suggesting the sound and reliable quality of data used. Of note, this epidemiological characteristic of age peak is different from that in other developed countries [21-24], where two-dose mumps vaccine has been routinely applied among children, which is usually outbreak among young adults. However, in China, the coverage of the second dose was relatively low, only $78.8 \%$ reported in some studies [25]. Besides, Liu and colleagues reported that waning immunity to mumps among infants who received one-dose vaccine during 18-24 months of age occurred with increasing age [26], resulting in the higher incidence rate in primary schools identified in the present study. These results suggest that improving the coverage of the second dose of vaccine should be prioritized to prevent mumps epidemics in China.
Besides, the third dose may be also necessary given the reemergence among adults in developed countries [21, 23].

Due to no routine vaccine for preventing HFMD [27], much more HFMD cases than mumps were reported in the present study. The incidence of HMFD showed an increasing tendency in China from 2008 to 2017 [9], but no corresponding increment of absolute case numbers was found in the present study. Again, we were not able to report the incidence rate which warranted further study and explanations. As was reported in the national report [9], the HFMD patients targeted on young infants aged less than 4 years old, accounting for $88 \%$ of the total cases. This population characteristic also explained that majority of the cases were found among children not in childcare center. In China, the childcare center usually accepts the infants aged 3 years above. Consequently, the development of strategies and programs for preventing and controlling such diseases should target on parents and caregivers.

To our knowledge, this is the first study to report that a fairly high percentage of patients had delays in hospital visiting for HFMD and mumps in China, as high as $43.1 \%$ for HFMD and $36.5 \%$ for epidemic parotitis, respectively. This delay in hospital visiting fails to make the timely notification to a local Center for Disease Control and Prevention, with extremely high probabilities of resulting in rapidly spreading within facilities and local outbreaks, even though majority of the cases had delayed durations of 2 to 3 days. Besides, the delay in hospital visiting and care at home might increase the risk of developing severe symptoms [28]. A similar situation was also reported in other infectious diseases like tuberculosis $[29,30]$. In addition, we found that 


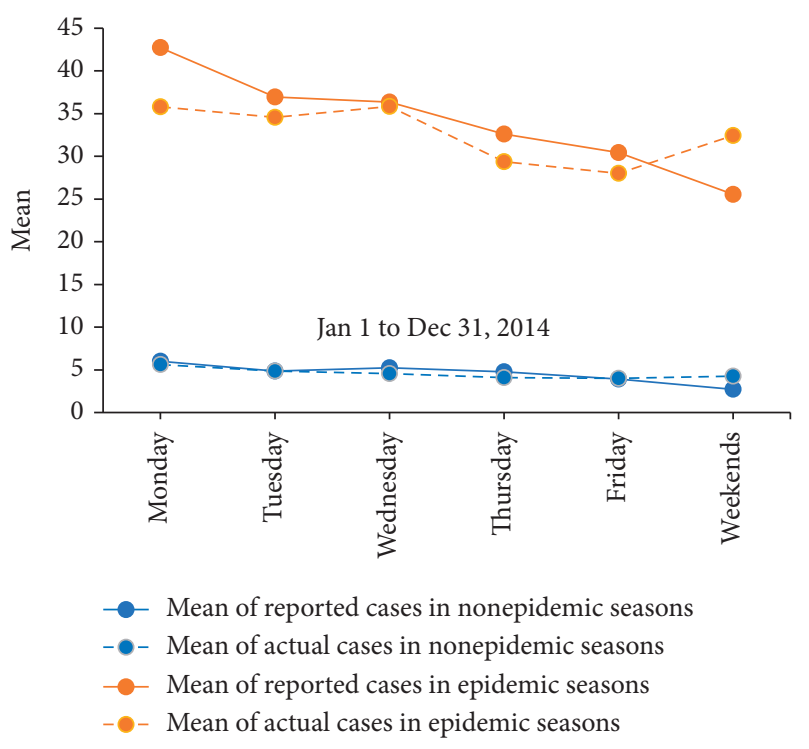

(a)

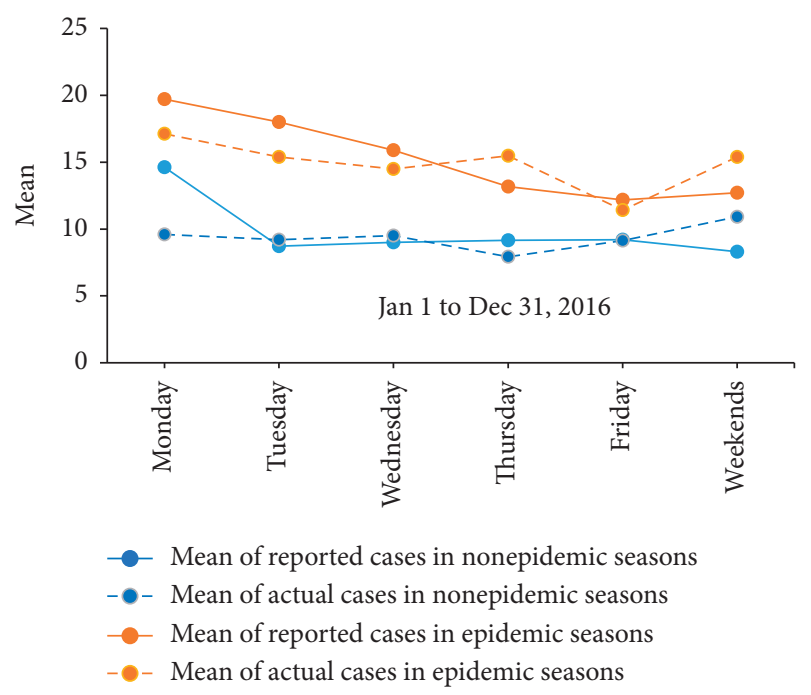

(c)

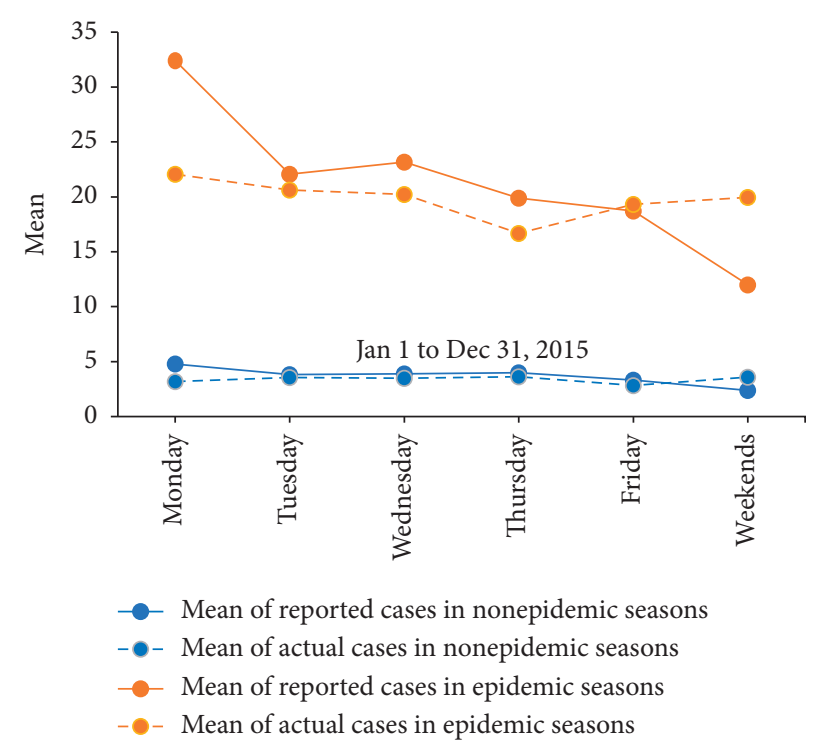

(b)

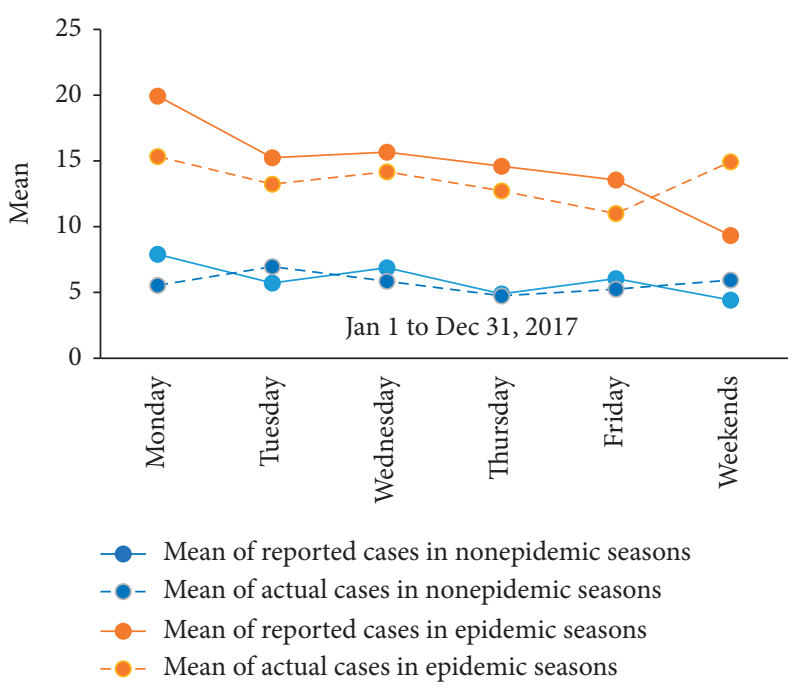

(d)

FIGURE 1: Distribution of weekly average actual cases and reported number of hand-foot-and-mouth disease cases between 2014 and 2017 in Hanzhong city, China.

patients occurred in nonepidemic seasons were more likely to experience the delay in hospital visiting, which implied that caregivers were less alert and might ignore the mild symptoms at the early stage of these diseases during nonepidemic seasons. We also found that children taken care at home were more likely to have delays in hospital visiting as compared with those in childcare center, where the morning and noon infectious disease inspection system was implemented and the staff were trained for screening and identifying the early signs, which suggested the necessities of improving related knowledge among caregivers. In the present study, it was also worthy to note that the risks of delay in hospital visiting for epidemic parotitis increased by years, which might be due to the decreasing of cases with typical clinical manifestations in recent years, especially among vaccinated populations [31]. In contrast, the incidence of HFMD kept at a much higher level that caused high concerns of all parties and strengthened the knowledge on prevention and treatment. This hypothesis was also supported by the finding that the risk of occurring delays in hospital visiting for HFMD kept steady in recent years. Taken together, given the fact that majority of cases have mild symptoms and that caregivers lack necessary knowledge on signs and symptoms at the early stage of these diseases, these results suggest the emphasis on community surveillance, prevention, and control especially in nonepidemic seasons [32]. Based on a community intervention trial, Guo and the coauthors reported that intensive education on handwashing for parents and caregivers of children aged 6 to 40 months in villages effectively reduced the incidence of HFMD [33], suggesting the feasibility and practicality of the community 


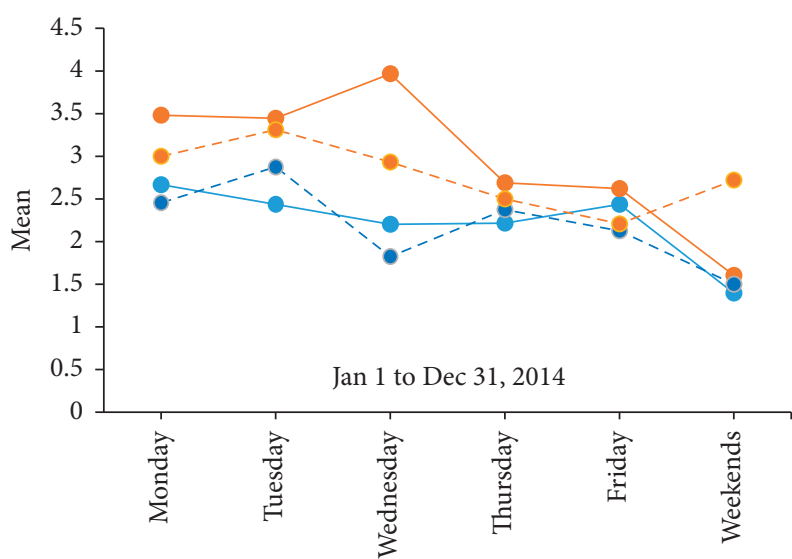

- - Mean of reported cases in nonepidemic seasons

- - Mean of actual cases in nonepidemic seasons

- Mean of reported cases in epidemic seasons

- - Mean of actual cases in epidemic seasons

(a)

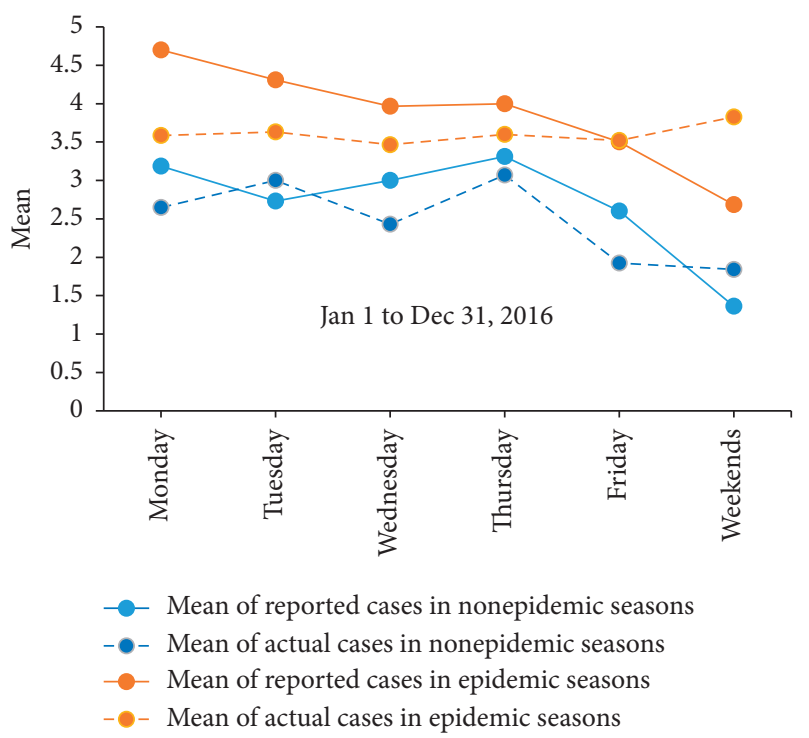

(c)
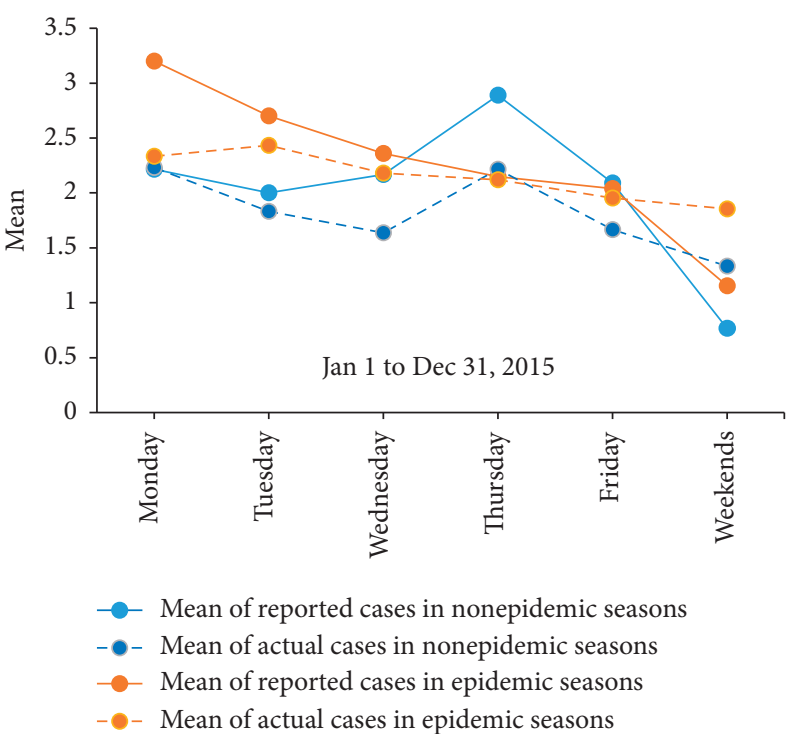

(b)

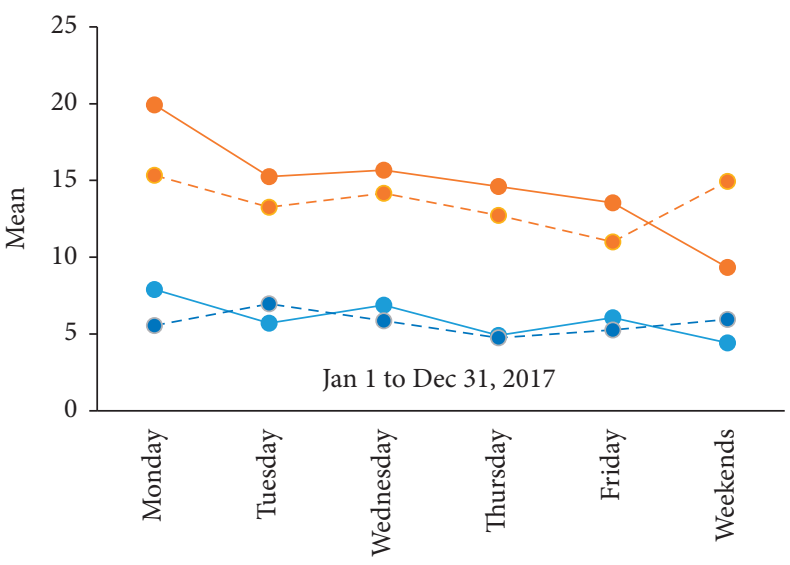

- Mean of reported cases in nonepidemic seasons

- Mean of actual cases in nonepidemic seasons

- Mean of reported cases in epidemic seasons

- Mean of actual cases in epidemic seasons

FIGURE 2: Distribution of weekly average actual cases and reported numbers of epidemic parotitis cases between 2014 and 2017 in Hanzhong city, China.

implementation for preventing these diseases and/or reducing the risk of delay in hospital visiting after disease onset.

In addition, we found that the online reports but not disease onset of cases showed a significant weekend effect for both HFMD and epidemic parotitis. Weekend effect is well noted in the context of hospital admissions of noncommunicable diseases such as emergency surgery and stroke, where patient has worse outcomes on weekend hospital admissions as compared with those on weekdays [34]. Nevertheless, the weekend effect is less studied in the infectious disease area, with a slightly different meaning in the present study, which indicates that the number of cases not reported on weekends is significantly higher than that on weekdays. As discussed in the delaying of hospital visiting above, these cases miss to report may lead to the local outbreak and severe symptoms, which warrants notice of related organizations. In terms of causes of weekend effect, two primary domains were investigated and discussed in the literature studies [35], patients themselves (parents and caregivers in the context of our study) and health system. Amirov and colleagues also reported that Mondays and Fridays had the highest occurrence of outbreak reporting in healthcare facilities from Toronto, Canada [36], and the authors concluded the health staff differences between weekend and weekdays as one of the causes. Although we were not able to determine the causes in the present study, health system might not be the factor in the causal link because we found that all cases were reported through the online disease surveillance system on the same day when 
patient visiting the hospital. Actually, to reduce deaths associated with the weekend effect, National Health Service in the UK implemented four priority clinical standards for a balanced seven-day hospital care. However, Meacock and Sutton did not find any significant changes in weekend mortality after three years' adoption of these clinical standards [37]. Besides, based on the data of unselected emergency admissions to four large Oxford hospitals between January 2006 and December 2014, Walker and colleagues adjusted for 15 common hematology and biochemistry test results not considered in other studies and found that these routine test results of patients substantially reduced excess mortality associated with emergency admission at weekends and public holidays [38]. Our finding that patients had delaying presenting to hospital visiting also indirectly supported this assumption. That said, a similar seven-day hospitalist program implemented in Taiwan showed potential for equaling the patient outcomes between weekday and weekend general medicine admissions, with a fairly smaller sample size of 861 [39]. Notwithstanding, from the perspective of health economy, Meacock and colleagues found that these programs were not cost-effective, i.e., the planned cost of implementing 7day services substantially exceeding the maximum amount that the National Health Service in the UK expected to eradicate the weekend effect [40]. Arguably, given the current evidence, patient-level differences rather than reduced health system and/or staffing levels play the key role in the context of weekend effect and are worthy to further examine the underlying mechanisms. For example, children playing activities not at home may increase the chances of contacts and being infected over the weekends, which consequently leads to the disease onset and more cases at the beginning of the week, resulting in the data artefact, i.e., fewer cases reported on weekends.

\subsection{Implications for Surveillance, Prevention, and Control of} Infectious Diseases. Recently in China, programs on public health education are continuously implemented and strengthened through various methods. However, it is difficult and challenging for the public and caregivers to know and master every piece of health knowledge due to the complicated and professional features of health knowledge. Consequently, we encouraged that the stratified and targeted education on infectious diseases that were common in children in corresponding ages should be developed and implemented in China, especially focusing on nonepidemic seasons and infectious diseases with low incidence rate. These strategies would effectively help caregivers master the corresponding prevention and control knowledge of infectious diseases such as early signs and thus improve the behavior of timely seeking medical service. Besides, these strategies also could help to reduce the detrimental effects of the weekend effects and ensure that the reported case numbers objectively indicated the actual status of disease incidence. In addition, when analyzing the surveillance data, the "weekend effect" should be considered using method like 7-day moving summation [17].

\section{Strengths and Limitations}

The surveillance data used in this study ensured the data quality and convincing results. However, this study has some limitations. First, we focused on the timing of the surveillance system but were unable to evaluate the accuracy and completeness of the surveillance data. As a result, the quality assessment of the entire surveillance system was not sufficiently thorough. Second, the data source did not allow us to examine the causes and/or consequences of the weekend effect that warrants further studies. Finally, we were unable to determine whether the disease was on outbreak that also may affect the quality assessment of the surveillance system.

\section{Conclusions}

The reported cases of HFMD and epidemic parotitis had an obvious weekend effect, with an increasing tendency of cases delaying in hospital visiting over the recent years in Hanzhong, China. Parents and caregivers rather than health systems should be primarily targeted for the prevention and control of infectious diseases and their local outbreaks such as community-based education on the second-dose vaccination of mumps and/or hand hygiene.

\section{Abbreviations}

HFMD: Hand-foot-and-mouth disease.

\section{Data Availability}

Data on HFMD and epidemic parotitis were obtained from the "Infectious disease report information management system" subsystem of the "China disease prevention and control information system."

\section{Consent}

The protocol was approved by Xi'an Jiaotong University Health Science Center and Center for Disease Control and Prevention, Hanzhong, Shaanxi, China.

\section{Conflicts of Interest}

The authors declare that they have no conflicts of interest.

\section{Authors' Contributions}

WJJ and ZLX conceived the topic. WJJ, ZZH, and QQ extracted and analyzed the data, interpreted results, and drafted the manuscript. ZLX supervised the study and revised the manuscript. All authors reviewed the manuscript and agreed to submit.

\section{Acknowledgments}

The authors thank the staffs of Hanzhong Center for Disease Control and Prevention for collecting the data. This work was supported by the National Natural Science Foundation of China (grant no. 81872633) and National Key Research 
and Development Program of China (grant nos. 2017YFC0907200 and 2017YFC0907201).

\section{Supplementary Materials}

Supplementary Table 1: weekly average of actual cases and reported numbers of epidemic parotitis between 2014 and 2017 in Hanzhong city, China. Supplementary Table 2: weekly average of actual cases and reported numbers of hand-foot-and-mouth disease between 2014 and 2017 in Hanzhong city, China. (Supplementary Materials)

\section{References}

[1] R. Jiang, Q. Yin, M. Xu et al., "Epidemiological characteristics of mumps in mainland China from 2004 to 2018 and key population for prevention and control," Zhongguo Dang Dai Er Ke Za Zhi, vol. 21, no. 5, pp. 441-444, 2019.

[2] J. Lu, Q. He, Y. Huang et al., "Epidemiological characteristics of mumps outbreaks in Guangzhou city from 2006-2018," Chinese Journal of School Health, vol. 40, no. 5, pp. 1-3, 2019.

[3] F. Bo, A. Cui, and X. Guo, "Analysis epidemiological and pathogen characters of mumps in China from 2004 to 2006," Chinese Journal of Vaccines and Immunization, vol. 15, no. 2, 2009.

[4] X. Yu, "Epidemiological features and prevention strategies of mumps in China," Modern Preventive Medicine, vol. 42, no. 15, pp. 2689-2691, 2015.

[5] J. Ma, L. Hao, H. Luo et al., "Analysis on epidemiological characteristics of mumps in China during 2008-2009," Chinese Journal of Vaccines and Immunization, vol. 16, no. 6, pp. 533-535, 2010.

[6] Y. Hu, L. Hao, and H. Wang, "Analysis on epidemiological characteristic of mumps in China, 2010-2012," Chinese Journal of Vaccines and Immunization, vol. 20, no. 2, pp. 127-131, 2014.

[7] J. Yang, "Epidemiologic characteristics of mumps in Liaocheng city from 2010-2012," Occupation and Health, vol. 30, no. 15, pp. 2144-2145, 2014.

[8] World Health Organization, WHO-recommended Surveillance Standard of Mumps, World Health Organization, Geneva, Switzerland, 2018.

[9] J. Zhang, "Trend of epidemics and variation of pathogens of hand, foot and mouth disease in China: a dynamic series analysis, 2008-2017," Zhonghua Liu Xing Bing Xue Za Zhi, vol. 40, no. 2, pp. 147-154, 2019.

[10] Y. Hu, G. Xiao, Y. Guo et al., "The epidemic features of the hand, foot, and mouth disease during 2008-2011 in China," Chinese Journal of Disease Control \& Prevention, vol. 18, no. 8, pp. 693-747, 2014.

[11] Q. Ding, Y. An, X. Ma et al., "Analysis on epidemiological characteristics of hand foot mouth disease in Hanzhong, Shaanxi, 2010-2015," Disease Surveillance, vol. 32, no. 1, pp. 34-37, 2017.

[12] S. Chadsuthi and S. Wichapeng, "The modelling of hand, foot, and mouth disease in contaminated environments in Bangkok, Thailand," Computational and Mathematical Methods in Medicine, vol. 2018, Article ID 5168931, 8 pages, 2018.

[13] L. Wang, F. Wang, S. Ding et al., "The weekend effect of handfoot-mouth disease reported in Shandong Province," Chinese Journal of Disease Control \& Prevention, vol. 19, no. 2, pp. 142-144, 2015.
[14] W. He, H. Fu, H. Tian et al., "The weekend effect of hand-footmouth disease reported in Baoji City," Journal of Public Health and Preventive Medicine, vol. 28, no. 2, pp. 42-44, 2017.

[15] W. Huang, R. Hong, and J. Ou, "Analysis of infectious disease surveillance quality between 2005 and 2013 in Fujian," Preventive Medicine Tribune, vol. 21, no. 3, pp. 220-223, 2015.

[16] J. Zhao, X. Su, Q. Guo et al., "Evaluation on communicable disease surveillance system," Disease Surveillance, vol. 32, no. 01, pp. 13-19, 2017.

[17] Y. Shi and J. Ma, "Application of exponential smoothing method in prediction and warning of epidemic mumps," Chinese Journal of Vaccines and Immunization, vol. 16, no. 3, pp. 233-237, 2010.

[18] Secretariat of Infectious Disease Professional Standards Committee TMOH, Compilation of the Current Statutory Report and Management Criteria for Infectious Disease (Health Industry Standard), Chinese Technical Publication, China, 2015.

[19] Q. R. Su, J. Liu, C. Ma et al., "Epidemic profile of mumps in China during 2004-2013," Zhonghua Yu Fang Yi Xue Za Zhi, vol. 50, no. 7, pp. 611-614, 2016.

[20] J. Sun, W. W. Yang, L. J. Zeng et al., "Surveillance data on notifiable infectious diseases among students aged 6-22 years in China, 2011-2016," Zhonghua Liu Xing Bing Xue Za Zhi, vol. 39, no. 12, pp. 1589-1595, 2018.

[21] J. A. Lewnard and Y. H. Grad, "Vaccine waning and mumps re-emergence in the United States," Science Translational Medicine, vol. 10, no. 433, 2018.

[22] A. I. Beleni and S. Borgmann, "Mumps in the vaccination age: global Epidemiology and the situation in Germany," International Journal of Environmental Research and Public Health, vol. 15, no. 8, 1618 pages, 2018.

[23] L. Veneti, K. Borgen, K. S. Borge et al., "Large outbreak of mumps virus genotype $G$ among vaccinated students in Norway, 2015 to 2016," Eurosurveillance, vol. 23, no. 38, 2018.

[24] A. Hviid, S. Rubin, and K. Mühlemann, "Mumps," The Lancet, vol. 371, no. 9616, pp. 932-944, 2008.

[25] J. P. Montgomery, Y. Zhang, B. Carlson, S. Ewing, X. Wang, and M. L. Boulton, "Measles vaccine coverage and series completion among children 0-8 Years of age in Tianjin, China," The Pediatric Infectious Disease Journal, vol. 34, no. 3, pp. 289-295, 2015.

[26] Y. Liu, Z. Liu, X. Deng et al., "Waning immunity of one-dose measles-mumps-rubella vaccine to mumps in children from kindergarten to early school age: a prospective study," Expert Review of Vaccines, vol. 17, no. 5, pp. 445-452, 2018.

[27] B. Cox and F. Levent, "Hand, foot, and mouth disease," Journal of the American Medical Association, vol. 320, no. 23, p. 2492, 2018.

[28] B. J. Sun, H. J. Chen, Y. Chen, X. D. An, and B. S. Zhou, "The risk factors of acquiring severe hand, foot, and mouth disease: a meta-analysis," Canadian Journal of Infectious Diseases and Medical Microbiology, vol. 2018, Article ID 2751457, 12 pages, 2018.

[29] T. Li, H. Zhang, H. D. Shewade, K. T. Soe, L. Wang, and X. Du, "Patient and health system delays before registration among migrant patients with tuberculosis who were transferred out in China," BMC Health Services Research, vol. 18, no. 1, p. 786, 2018.

[30] X. J. Wang, Q. Fu, Z. B. Zhang et al., "Delay on care-seeking and related influencing factors among tuberculosis patients in Wuhan, 2008-2017," Zhonghua Liu Xing Bing Xue Za Zhi, vol. 40, no. 6, pp. 643-647, 2019. 
[31] Q. R. Su and K. H. Yao, "Epidemiological and clinical features of vaccine-preventable diseases in vaccine era," Zhongguo Dang Dai Er Ke Za Zhi, vol. 21, no. 3, pp. 195-198, 2019.

[32] J. H. Chan, C. K. Law, E. Hamblion et al., "Best practices to prevent transmission and control outbreaks of hand, foot, and mouth disease in childcare facilities: a systematic review," Hong Kong Medical Journal, vol. 23, no. 2, pp. 177-190, 2017.

[33] N. Guo, H. Ma, J. Deng et al., "Effect of hand washing and personal hygiene on hand food mouth disease: a community intervention study," Medicine, vol. 97, no. 51, Article ID e13144, 2018.

[34] Y. F. Chen, X. Armoiry, C. Higenbottam et al., "Magnitude and modifiers of the weekend effect in hospital admissions: a systematic review and meta-analysis," BMJ Open, vol. 9, no. 6, Article ID e025764, 2019.

[35] M. McKee, "The weekend effect: now you see it, now you don't," British Medical Journal, vol. 353, Article ID i2750, 2016.

[36] C. Amirov, R. N. Walton, S. Ahmed, M. A. Binns, J. E. Van Toen, and H. L. Candon, "Distribution of outbreak reporting in health care institutions by day of the week," American Journal of Infection Control, vol. 40, no. 10, pp. 979-982, 2012.

[37] R. Meacock and M. Sutton, "Elevated mortality among weekend hospital admissions is not associated with adoption of seven day clinical standards," Emergency Medicine Journal, vol. 35, no. 2, pp. 108-113, 2018.

[38] A. S. Walker, A. Mason, T. P. Quan et al., "Mortality risks associated with emergency admissions during weekends and public holidays: an analysis of electronic health records," The Lancet, vol. 390, no. 10089, pp. 62-72, 2017.

[39] N. C. Hsu, C. C. Huang, C. C. Shu, and M.-C. Yang, "Implementation of a seven-day hospitalist program to improve the outcomes of the weekend admission: a retrospective before-after study in Taiwan," PLoS One, vol. 13, no. 3, Article ID e0194833, 2018.

[40] R. Meacock, T. Doran, and M. Sutton, "What are the costs and benefits of providing comprehensive seven-day services for emergency hospital admissions?" Health Economics, vol. 24, no. 8, pp. 907-912, 2015. 